\title{
Towards Ultrahigh Speed Impulse Radio THz Wireless Communications
}

\author{
Yu, Xianbin; Galili, Michael; Morioka, Toshio; Jepsen, Peter Uhd; Oxenløwe, Leif Katsuo
}

Published in:

Proceedings of ICTON 2015

Link to article, DOI:

10.1109/icton.2015.7193623

Publication date:

2015

Document Version

Peer reviewed version

Link back to DTU Orbit

Citation $(A P A)$ :

Yu, X., Galili, M., Morioka, T., Jepsen, P. U., \& Oxenløwe, L. K. (2015). Towards Ultrahigh Speed Impulse Radio THz Wireless Communications. In Proceedings of ICTON 2015 [7193623] IEEE.

https://doi.org/10.1109/icton.2015.7193623

\section{General rights}

Copyright and moral rights for the publications made accessible in the public portal are retained by the authors and/or other copyright owners and it is a condition of accessing publications that users recognise and abide by the legal requirements associated with these rights.

- Users may download and print one copy of any publication from the public portal for the purpose of private study or research.

- You may not further distribute the material or use it for any profit-making activity or commercial gain

- You may freely distribute the URL identifying the publication in the public portal 


\title{
Towards Ultrahigh Speed Impulse Radio THz Wireless Communications
}

\author{
Xianbin Yu, Michael Galili, Toshio Morioka, Peter Uhd Jepsen, and Leif K. Oxenløwe \\ DTU Fotonik, Department of Photonics Engineering, Technical University of Denmark, \\ DK-2800, Kgs. Lyngby, Denmark, e-mail: xiyu@fotonik.dtu.dk
}

\begin{abstract}
$\mathrm{THz}$ impulse radio technologies promise a new paradigm of fast wireless access with simplified wireless reception. However, huge loss of propagating broad bandwidth $\mathrm{THz}$ impulse radio signals limits $\mathrm{THz}$ wireless transmission distance and reduces the achievable link data rates. In this paper, we evaluate the realistic throughput and accessible wireless range of a $\mathrm{THz}$ impulse radio communication link based on a uni-travelling photodiode (UTC-PD) as THz emitter and a photoconductive antenna (PCA) as THz receiver. The impact of highly frequency-selective $\mathrm{THz}$ channel and the noise in the system are also considered.
\end{abstract}

Keywords: THz photonics, THz wireless communication, impulse radio, photoconductive sampling.

\section{INTRODUCTION}

$\mathrm{THz}$ technologies have been widely recognized as next frontier for developing ultrafast wireless communication [1][2], aiming at accommodating up to Terabit-per-second (Tbps) wireless data rates in 2020. The most challenging aspects are obviously the development of efficient ultra-broadband components operating in the $\mathrm{THz}$ frequency bands, particularly for generating and detecting $\mathrm{THz}$ communication signals. Up to date, a lot of efforts have been put on developing the photonic devices, utilizing the extremely fast photoresponse and hence overcoming the bandwidth limitation in the electronic techniques. More specifically, uni-travelling carrier photodiodes (UTC-PDs) have been of great interest in opto-electronic generation of THz signals due to the very large bandwidth and high THz conversion efficiency [3]. The most recently developed UTC-PD has exhibited a bandwidth up to $2.5 \mathrm{THz}[4]$.

From the viewpoint of a communication system, the impressive performance of the UTC-PDs accelerates the development of high speed photonic wireless communications. The UTC-PDs have indeed been widely used in the demonstrated millimeter-wave and sub-THz wireless systems [5]-[17]. Figure 1 summarizes recent contributions on progressing high speed photonic wireless communication systems, in the frequency range of $100 \mathrm{GHz}-600 \mathrm{GHz}$ [7]-[17]. These systems operate in different narrow frequency windows, and thus suffer less loss from the atmospheric propagation. However, there is only tens of $\mathrm{GHz}$ bandwidth available for each single frequency window, which makes narrow band carrier modulation technique difficult to approach Tbps, as minimum $200 \mathrm{GHz}$ bandwidth will be required to obtain a reasonable signal-to-noise ratio for a Tbps signal. To further increase the throughput of a $\mathrm{THz}$ communication system, most attentions are so far paid to apply multidimensional modulation schemes (amplitude modulation, phase modulation, frequency multiplexing, time multiplexing and polarization multiplexing). It noted the increasing of $\mathrm{THz}$ spectral efficiency requires higher linearity and larger spur free dynamic range.

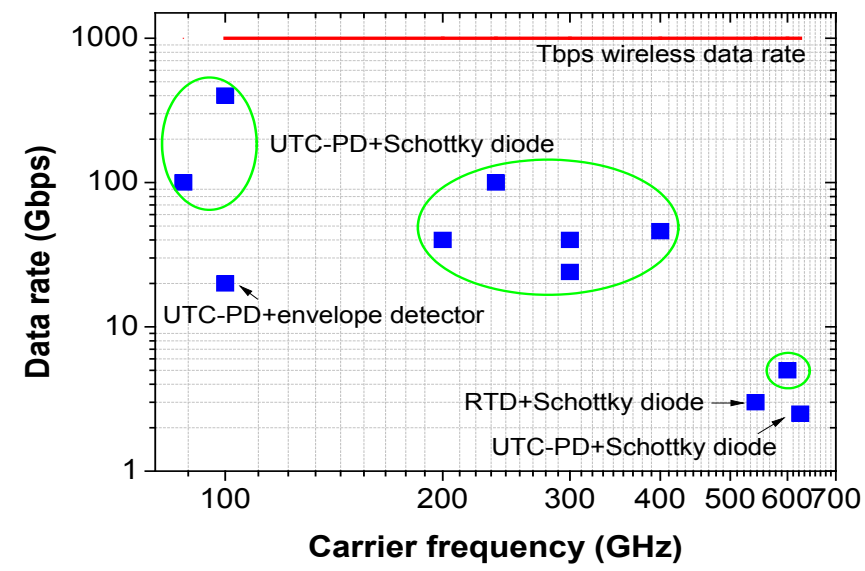

Figure 1. Reported high speed sub-THz/THz carrier modulation communication systems.

Alternatively, a THz impulse radio (IR) communication, similar to impulse radio ultra-wideband systems $(3.1-10.6 \mathrm{GHz})[18][19]$, has also the potential of supporting very large capacity at a scale of Tbps within a small wireless coverage $(<1 \mathrm{~m})$ [20][21], by exploring an extremely large RF frequency band for communication. In addition, a pulsed system features some other advantages, e.g. larger bandwidth (simple modulation scheme), easily recoverable distortion and less noise effect. At the receiver side, low-temperature- 
grown GaAs photoconductive antennas (PCAs) can be an option, as they typically consume sub-nJ energy per pulse and can handle sufficiently large bandwidth in the UTC-PD. The capacity of a THz impulse radio communication link has been theoretically analyzed in [20], but the realistically achievable link throughput relies on the performance of $\mathrm{THz}$ components employed and reception scheme, and this is not yet investigated.

In this paper, we will estimate the realistic throughput and accessible wireless range of a $\mathrm{THz}$ impulse radio wireless communication link by employing a UTC-PD as THz emitter and a photoconductive antenna as THz receiver. The link performance will be reasonably analyzed by taking into account the impact of THz frequencyselective channel properties and the noise in the system.

\section{THz IMPULSE RADIO SYSTEMS AND PERFORMANCE ANALYSIS}

\subsection{THz impulse radio systems}

The schematic THz impulse radio communication system under consideration in this paper is shown in Fig. 2. A time-multiplexed ultrashort optical pulse train at very high data rates well beyond $100 \mathrm{Gbps}$ is launched into a $\mathrm{THz}$ emitter. The illumination of each optical ultrashort pulse at the emitter correspondingly generates a $\mathrm{THz}$ pulse, and then radiated by an antenna integrated in the $\mathrm{THz}$ emitter. After free space transmission, the $\mathrm{THz}$ pulse train is wirelessly received by a $\mathrm{THz}$ receiver. In order to release bandwidth requirements in receiving such an ultrahigh speed signal, we will consider time demultiplexing technique in the receiver. A sampling pulsed laser at a relatively low repetition rate (e.g. $10 \mathrm{GHz}$ ) is used to demultiplex ultrafast $\mathrm{THz}$ pulse train into the base rate of sampling laser. In our system, we will consider an ultra-broadband UTC-PD and a PCA as the THz emitter and receiver, respectively. Both of these two components are commercially available and commonly used in $\mathrm{THz}$ systems.

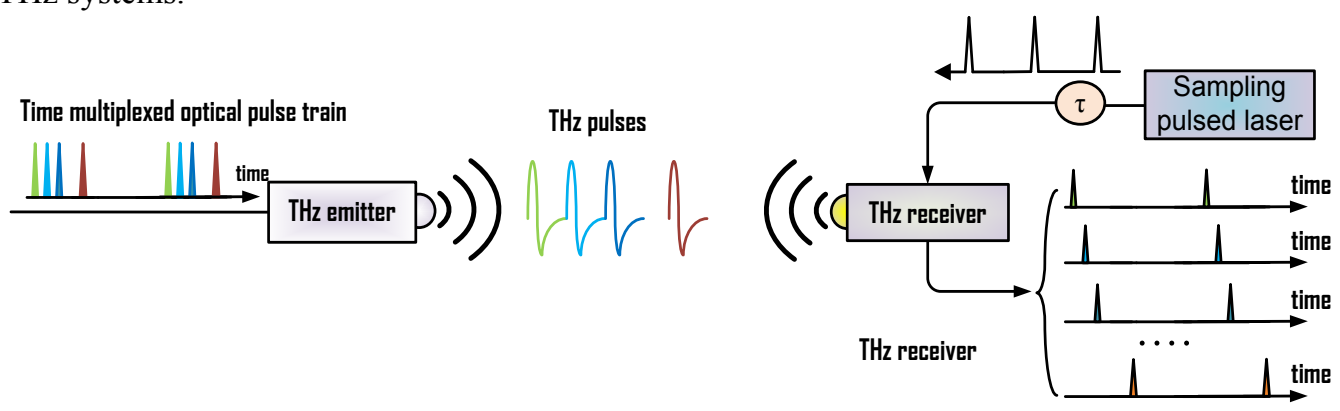

Figure 2. Concept of an ultrafast THz impulse radio communication link employing optical time multiplexing at the transmitter and time demultiplexing at the receiver. THz emitter: UTC-PD, THz receiver: PCA.

\subsection{Noise in the system}

There are several major noise sources in the THz communication system above, and molecular absorption noise in the wireless channel and thermal noise/shot noise in the UTC-PD are dominant, amongst others. When the molecular absorption is considerably high in the system, the internal vibration of the molecules turns into the emission of $\mathrm{THz}$ radiation at the same frequency that the incident waves that provoked this motion, which can be considered as a noise factor [20] and estimated by

$$
\sigma_{m o l}^{2}(f, d)=k_{B} T \cdot \int_{B}\left[1-e^{k(f) d}\right] d f
$$

where $k(f)$ denotes the absorption strength, the absorption coefficient at the frequency component $f$, $d$ is wireless distance, $T$ is the operating environment temperature of the system, $k_{B}$ is the Boltzmann constant, and $B$ is the effective noise bandwidth.

The shot noise is dependent on the average photocurrent $I_{\text {avg }}$ in the photodiode and the thermal noise does not.

$$
\begin{aligned}
& \sigma_{\text {shot }}^{2}=2 q R P_{\text {avg }} B \\
& \sigma_{\text {thermal }}^{2}=4 k_{B} T / R_{l} \cdot B
\end{aligned}
$$

Here $q$ is the electron charge, $R$ is the responsivity of the UTC-PD, $P_{a v g}$ is the average optical power and $R_{l}$ is the load resistor. The total noise in the system will be

\subsection{Performance analysis}

$$
\sigma_{\text {total }}^{2}=\sigma_{\text {mol }}^{2}+\sigma_{\text {shot }}^{2}+\sigma_{\text {thermal }}^{2}
$$

In our numerical analysis, a commercially available UTC-PD with a $3 \mathrm{~dB}$ bandwidth of $310 \mathrm{GHz}$ and a DC responsivity of $0.25 \mathrm{~A} / \mathrm{W}$ is considered. The system performance is estimated by analyzing the performance of demultiplexed $10 \mathrm{GHz}$ signals after free space wireless propagation, in terms of wireless distance-dependent signal to noise ratio and $\mathrm{Q}$ factor. The wireless propagation is considered in an office- or lab-based indoor environment $\left(20^{\circ} \mathrm{C}\right.$ and $51 \%$ relative humidity). The PCA considered here features very fast photoresponse 
which is governed by its trapping time ( $0.7 \mathrm{fs})$ and trap emptying time (10 fs). The average power launching into the UTC-PD and the PCA are respected to be below their thermal damage thresholds, and they are $20 \mathrm{~mW}$ for the $\mathrm{THz}$ emitter and $30 \mathrm{~mW}$ for the $\mathrm{THz}$ receiver, respectively. The strength of the absorption by a specific type of molecules is directly obtained from the Jet Propulsion Laboratory (JPL) database.

Figure 3(a) shows $640 \mathrm{Gbps}$ THz IR signal generated from 100 fs ultrashort optical pulse train and the impact of wireless propagation on the waveform of $\mathrm{THz}$ pulses. We can see that each optical pulse individually generates a THz pulse. The emitted THz pulses are monocycle-like, because THz radiation in a dipole or bow-tie antenna is proportional to the derivative of the instantaneous current flowing through the antenna gap. It is noted that noise in the system is not included, but we can observe that the $\mathrm{THz}$ pulses after $1 \mathrm{~m}$ wireless distance exhibits noise-like background in the time domain, which apparently degrades the received signal to noise ratio. This is because the ultrashort $\mathrm{THz}$ pulse is extremely broadband in the frequency domain and the wireless propagation channel is highly selective, particularly in the frequency components above $1 \mathrm{THz}$.

The quality of $640 \mathrm{Gbps} \mathrm{THz}$ impulse radio signals is evaluated by calculating $\mathrm{Q}$ factor of wirelessly received $10 \mathrm{GHz}$ pulses. Taking into account the noise in the system, the demultiplexed $10 \mathrm{GHz} \mathrm{THz}$ pulse trains after $0.1 \mathrm{~m}$ wireless propagation is presented in Fig. 3(b), and the SNR in this case is $22 \mathrm{~dB}$, which can be expected to achieve a $\mathrm{Q}$ factor of 10.6. The wireless distance dependent $\mathrm{Q}$ factor for demultiplexed $10 \mathrm{GHz}$ signals is shown in Fig. 3(c). We can see that within the wireless range of $<0.5 \mathrm{~m}$, performance of below the forward error correction (FEC, bit-error rate (BER) of 2e-3) can be achieved.

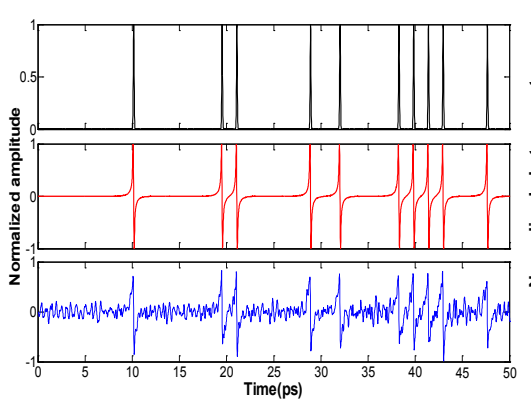

(a)

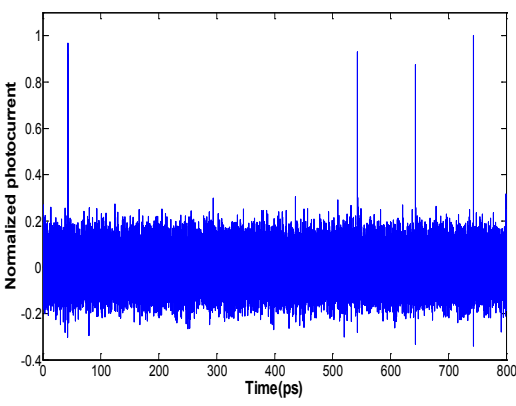

(b)

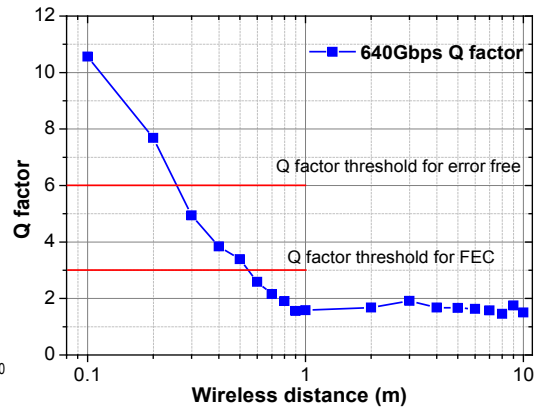

(c)

Figure 3: (a) $640 \mathrm{Gbps} 100 \mathrm{fs}$ optical pulse train with on-off keying modulation (above), $640 \mathrm{Gbps} \mathrm{THz}$ radiation (middle) and wireless propagation over $1 \mathrm{~m}$ (bottom) without taking into account noise in the system; (b) De-multiplexed $10 \mathrm{GHz}$ pulse train after $0.1 \mathrm{~m}$ wireless; (c) BER performance vs wireless distance.

\section{CONCLUSIONS}

$\mathrm{THz}$ impulse radio technology explores extremely broadband RF frequency for communications and has a high potential of realizing ultrahigh speed wireless data rates. We have evaluated the realistic throughput and accessible wireless range of a $\mathrm{THz}$ impulse radio communication link based on commercially available $\mathrm{THz}$ components, an UTC-PD as THz emitter and a PCA as THz receiver. $640 \mathrm{Gbps} \mathrm{THz}$ impulse radio throughput can be supported in room temperature environment in a small cell range $(<0.5 \mathrm{~m})$ by employing time demultiplexing scheme for receiver. The limited access range is due to huge loss and additional noise caused by the THz propagation channel. Therefore, impulse radio Tbps $\mathrm{THz}$ communication would find applications in nanocell backhaul, short range interconnections, superfast board-to-board communication, and some particular cases of wireless delivering large volume of data (e.g. E-healthcare images).

\section{ACKNOWLEDGEMENTS}

This research was supported by the ERC-PoC project TWIST within the European Union's Horizon 2020 research and innovation programme. The authors also would like to thank NTT in Japan for supplying an UTC-PD.

\section{REFERENCES}

[1] F. Akyildiz et al:: Terahertz band: Next frontier for wireless communications, J. Physical Commun., pp. 1632, 2014.

[2] X. Yu et al.: The prospects of ultra-broadband THz wireless communications, in Proc. ICTON 2014, paper Th.A3.3.

[3] T. Nagatsuma et al.: Gigabit wireless link using 300-400 GHz bands, MWP 2009, Paper Th2.3.

[4] T. Ishibashi et al.: Continuous THz wave generation by photodiodes up to $2.5 \mathrm{THz}$, in Proc. IRMMW 2013, Germany.

[5] L. Deng et al.: Fiber wireless transmission of 8.3Gb/s/ch optical QPSK-OFDM W-band (75-110 GHz) signals, IEEE Photon. Techno. Lett., pp. 383-385, 2012. 
[6] X. Pang et al.: 25Gbit/s QPSK hybrid fiber-wireless transmission in the W-Band (75-110 GHz) with remote antenna unit for in-building wireless networks, IEEE Photonics Journal, vol. 4, no. 3, pp. 691-698, 2012.

[7] X. Pang et al.:100Gbit/s hybrid optical fiber-wireless link in the W-band, (75-110 GHz), Opt. Exp., vol. 19, 2011.

[8] J.-W. Shi et al.: Millimeter-wave photonic wireless links for very-high data rate communication, NPG Asia Mater., pp. 41-48, 2011.

[9] X. Li et al.: A 400G optical wireless integration delivery system, vol. 21, no. 16, Opt. Exp., 2013.

[10] A. J. Seeds et al.: Terahertz photonics for wireless communications, J. Lightwave Techno., pp. 579-587, 2015.

[11] S. Koenig et al.: Wireless sub-THz communication system with high data rate, Nature Photon., 2013.

[12] H. J. Song et al.: $24 \mathrm{Gbit} / \mathrm{s}$ data transmission in $300 \mathrm{GHz}$ band for future terahertz communications, Electron. Lett., pp. 953-954, 2012.

[13] T. Nagatsuma et al.: Terahertz wireless communications based on photonics technologies, Opt. Exp. pp. 23736-23747, 2013.

[14] G. Ducournau et al.: Ultrawide-bandwidth single-channel 0.4-THz wireless link combining broadband quasi-optic photomixer and coherent detection, IEEE Trans. THz Sci. Technol., pp. 328-337, 2014.

[15] K. Ishigaki et al.: Direct intensity modulation and wireless data transmission characteristics of terahertzoscillating resonant tunnelling diodes, Electron. Lett., pp. 582-583, 2012.

[16] G. Ducournau et al.: High-definition television transmission at $600 \mathrm{GHz}$ combining $\mathrm{THz}$ photonics hotspot and high-sensitivity heterodyne receiver, Electron. Lett., 50, 5, pp. 413-415, 2014.

[17] L. Moeller et al.: 2.5Gbit/s duobinary signalling with narrow bandwidth 0.625 terahertz source, Electron. Lett., pp 856-858, 2011.

[18] X. Yu et al.: System wide implementation of photonically generated impulse radio ultra-wideband for gigabit fiber-wireless access, J. Lightwave Techno., pp. 264-275, 2013.

[19] X. Yu et al.: Distribution of photonically generated 5Gbps impulse radio UWB signals over fiber, Optics Lett., pp. 810-812, 2011,.

[20] J. M. Jornet et al.: Channel capacity of electromagnetic nanonetworks in the terahertz band, ICC Communications, 2010.

[21] Y. Yang et al.: Understanding THz pulse propagation in the atmosphere, THz Science Techno., 2012. 